Pacific Journal of Mathematic 


\title{
QUASI DIMENSION TYPE. II. TYPES IN 1-DIMENSIONAL SPACES
}

\author{
JACK SEGAL
}

In this paper we consider the partial ordering induced on 1-dimensional continua by quasi dimension type. For example, we show that the pseudo-arc precedes every snake-like continuum in this partial ordering. We also obtain sufficient conditions in terms of quasi dimension type for the embedding of Peano continua in the plane. Finally, necessary and sufficient conditions in terms of quasi dimension type are given for a continuum to be tree-like and to be 1-dimensional.

All spaces considered in this paper are metric. The distance between two points $x, y$ will be denoted by $\rho(x, y)$ and the diameter of a set $A$ will be denoted by $\delta(A)$. By "covering" we mean "open covering". We use $d X$ and $q X$ for the Fréchet and quasi dimension types of $X$ respectively. Recall that $d X \leqq d Y$ provided there is a homeomorphism of $X$ into $Y ; q X \leqq q Y$ provided that for each covering $\alpha$ of $X$ there is a closed $\alpha$-mapping of $X$ into $Y$. (A continuous function $f: X \rightarrow Y$ is an $\alpha$-map if there is a covering $\beta$ of $Y$ such that $f^{-1}[\beta]$ refines $\alpha$ and $f$ is closed if it takes closed subsets of $X$ into closed subsets of $Y$.) A compact connected space of more than one point will be called a continuum. A chain is a finite collection of open sets $U_{1}, U_{2}, \cdots, U_{n}$ such that $U_{i}$ intersects $U_{j}$ if $i=j-1, j$, $j+1$, and otherwise $\rho\left(U_{i}, U_{j}\right)>0$. If the links of a chain are of diameter less than $\varepsilon$, the chain is called an $\varepsilon$-chain. A continuum $X$ is called snake-like if for each $\varepsilon>0$ it can be covered by an $\varepsilon$-chain (whose links are subsets of $X$ ). Finally, $S_{(x, \varepsilon)}=\{y \mid \rho(x, y)<\varepsilon\}, R^{n}$ denotes Euclidean $n$-space and $J$ denotes the positive integers.

1. Quasi dimension types of snake-like continua. Bing [1] proved that any two snake-like hereditarily indecomposable continua are homeomorphic. Such continua are called pseudo-arcs and throughout this paper we use $\psi$ to denote a pseudo-arc.

Lemma 1.1. [22, Th. 6]. If, for each $\varepsilon>0$, a continuum can be e-mapped onto a hereditarily indecomposable continuum, then $X$ is hereditarily indecomposable.

Lemma 1.2. If, for each $\varepsilon>0$, a continuum $X$ can be $\varepsilon$-mapped onto a snake-like continuum, then $X$ is snake-like. 
THEOREM 1.1. If $X$ is not totally disconnected and $q X \leqq q \psi$, then $q X=q \psi$.

Thus we have that no nontotally disconnected space precedes $\psi$ and hence in this sense $\psi$ is minimal for those nontotally disconnected spaces with which it is comparable. We will now consider how large this class of spaces is. Certainly, for any snake-like continuum $X$ which contains an arc or a pseudo-arc, we have $q \psi \leqq q X$. However, this does not exhaust the class of snake-like continua. Bing [3], Lelek [17], and others have shown that $\psi$ can be mapped onto any snake-like continuum. We sharpen this result by proving that, for any $\varepsilon>0, \psi$ can be $\varepsilon$-mapped onto any snake-like continuum. Furthermore, since for any $\varepsilon>0$, an arc can be $\varepsilon$-mapped onto any Peano space $X$ which contains an open $n$-cell, $n \geqq 2$, as an open set (see [16]) we may conclude that $\psi$ can be $\varepsilon$-mapped onto $X$ (so that $q \psi \leqq q X)$. But since we need only $\varepsilon$-mappings of $\psi$ into a space, we have $q \psi \leqq q X$ if $X$ contains a snake-like continuum. So $\psi$ occupies a position with respect to spaces which have snake-like subcontinua similar to that of the Cantor set with respect to all infinite subsets of the line.

THEOREM 1.2. If $X$ is a snake-like continuum and $\varepsilon>0$, then there is an e-mapping of $\psi$ onto $X$ (and so $q \psi \leqq q X$ ).

Proof. To obtain the desired result we modify Bing's proof [3, p. 446] that $\psi$ can be mapped onto $X$. For any $\varepsilon>0$, let $D_{1}, D_{2}, \cdots$ be a sequence such that $D_{i}$ is a $(\varepsilon / 2 i)$-chain covering $X$ and $D_{i+1}$ is a refinement of $D_{i}$. It follows from Theorem 7 of [4] that there is a sequence of coverings $E_{1}, E_{2}, \cdots$ of $\psi$ such that: (1) $E_{i}$ is a $(\varepsilon / 2 i)$ chain covering $\psi,(2) E_{i}$ has the same number of links as $D_{i}$ and (3) for the $j$ th link of $D_{i+1}$ there is an integer $n(i, j)$ such that the $j$ th link of $D_{i+1}$ lies in the $n(i, j)$ th link of $D_{i}$ and the $j$ th link of $E_{i+1}$ lies in the $n(i, j)$ th link of $E_{i}$.

For each point $p$ of $\psi$ let $e(p, i)$ be the union of the links of $E_{i}$ containing $p$ and $d(p, i)$ be the union of the corresponding links of $D_{i}$. Note that $e(p, i+1) \subset e(p, i)$ and $d(p, i+1) \subset d(p, i)$. For each point $p$ of $\psi$ let $f_{\varepsilon}(p)$ be the intersection of the closures of the $d(p, i)$ 's. Then $f_{\varepsilon}$ is a mapping of $\psi$ onto $X$ and we need show that it is an $\varepsilon$-mapping.

Suppose $f_{\varepsilon}(p)=f_{\varepsilon}(q)$. Then $d(p, 2) \cap d(q, 2) \neq \varnothing$ and this implies that two adjacent links of $D_{2}$, say $d_{j}^{2}$ and $d_{k}^{2}$ (where $k=j-1, j$ or $j+1)$, are such that

(4) $f_{\varepsilon}(p) \in d_{j}^{2} \cap d_{k}^{2}$ (which implies $f_{\varepsilon}(p) \in d_{n(1, j)}^{1} \cap d_{n(1, k)}^{1}$ ) and

( 5 ) $\quad d_{j}^{2} \subset d(p, 2)$ and $d_{k}^{2} \subset d(q, 2)$. 
Therefore, from (4) we get

(6) $e_{n(i, j)}^{1} \cap e_{n(i, k)}^{1} \supset e_{j}^{2} \cap e_{k}^{2} \neq \varnothing$

and from (5) we get

( 7 ) $p \in e_{j}^{2}$ and $q \in e_{k}^{2}$.

So we have $e(p, 1) \cap e(q, 1) \neq \varnothing$ and it follows that

$$
\rho(p, q) \leqq \delta(e(p, 1))+\delta(e(q, 1))<\varepsilon / 2+\varepsilon / 2=\varepsilon .
$$

This yields the desired result that $f_{\varepsilon}$ is an $\varepsilon$-mapping.

THEOREM 1.2'. If $X$ is a space which contains a snake-like continuum, then $q \psi \leqq q X$.

We now show that there is a planar continuum $M$ (originally described by Whyburn [24] as an example of a continuum each subcontinuum of which separates the plane) such that $q \psi \leqq q M$ is false. By Theorem 1.1 this means that $\psi$ and $M$ are not comparable. What we actually prove is that no subcontinuum of $M$ is the continuous image of $\psi$.

The continuum $M$ has a rather complicated description but we can obtain our desired result by using only some of its properties. First note that $M$ arises in a condensation process from a ray spiralling on a circle. Letting $A$ denote the latter continuum it is not difficult to see that $A$ is the continuous image of $M$. Therefore $\psi$ cannot be mapped onto $M$; otherwise, we could map $\psi$ onto $A$ and this is impossible (see [17, Example 2]). Furthermore, Whyburn shows that (1) every subcontinuum of $M$ contains a continuum which is homeomorphic to $M$ and (2) if any subcontinuum $N$ of $M$ contains a point of a subcontinuum $W$ of $M$ which is homeomorphic to $M$, then $N$ contains $W$ or $W$ contains $N$. We use $C(X)$ to denote the space of all nonvacuous subcontinua of a continuum $X$ metrized by the Hausdorff metric (see [14]). If $Y$ is a closed subset of $X$, then $C(X, Y)$ denotes the subset of $C(X)$ consisting of continua which contain $Y$.

THEOREM 1.3. The continuum $M$ has no subcontinuum which is the continuous image of pseudo-arc $\psi$.

Proof. Suppose $M$ properly contains a continuum $X$ which is the continuous image of $\psi$. Say $f$ is a mapping of $\psi$ onto $X$. Then $X$ contains a continuum $W$ which is homeomorphic to $M$. Suppose $x \in W$. Then there is a $y \in \psi$ such that $f(y)=x$. Let $B=\left\{B_{t} \mid 0 \leqq t \leqq 1\right\}$ be the unique arc in $C(\psi)$ from $\{y\}$ to $\psi$ with $B_{0}=\{y\}$ and $B_{1}=\psi$ (see [14, Th. 8.4]). Let $f^{\prime}: C(\psi) \rightarrow C(X)$ be defined by $f^{\prime}(Y)=f[Y]$ for each $Y \in C(\psi)$. Then $f^{\prime}$ is a mapping. Denote $f^{\prime}\left(B_{t}\right)$ by $B_{t}^{\prime}$ and $f^{\prime}[B]$ 
by $B^{\prime}$. Then $B^{\prime}$ is a subcontinuum of $C(X)$ which contains $X$ and $\{x\}$. Now since $B_{t}^{\prime} \cap W \neq \varnothing$ by property (2) of $M$ we have $B_{t}^{\prime}$ contains $W$ or $W$ contains $B_{t}^{\prime}$ for $0 \leqq t \leqq 1$. However, since $M$ is not the continuous image of $\psi$ neither is $W$ so that $B_{t}^{\prime}$ properly contains $W$ or $W$ properly contains $B_{t}^{\prime}$. Therefore

$$
B_{t}^{\prime} \in C(X, W) \cup C(W), \quad \text { for } 0 \leqq t \leqq 1
$$

and so

$$
B^{\prime} \subset C(X, W) \cup C(W) \text {. }
$$

It is possible to define a real-valued continuous function, $\mu$, of $C(X)$ onto the unit interval $I$ (see [23]) with the properties:

(1) If $Y_{1} \subset Y_{2}, Y_{1} \neq Y_{2}$ then $\mu\left(Y_{1}\right)<\mu\left(Y_{2}\right)$.

(2) $\mu(X)=1$, and for any $x \in X, \mu(\{x\})=0$.

Therefore since $B^{\prime}$ contains $\{x\}$ and $X$ we have that the continuum $\mu\left[B^{\prime}\right]$ contains 0 and 1 and so $\mu\left[B^{\prime}\right]=I$. Thus there is $t_{0}, 0<t_{0}<1$, such that $\mu\left(B_{t_{0}}^{\prime}\right)=\mu(W)$. Now in $C(X, W) \cup C(W)$ the only element with $\mu$-value equal to $\mu(W)$ is $W$ itself since all other elements either are properly contained or properly contain $W$ and (1) applies. So finally we have $B_{t_{0}}^{\prime}=W$ which is a contradiction.

CoROllary 1.3. The quasi dimension types of the pseudo-arc $\psi$ and Whyburn's continuum $M$ are not comparable.

Question. Are there snake-like continua whose quasi dimension types are not comparable?

Question. What is the cardinality of the set of quasi dimension types of snake-like continua?

Lemma 1.3. Suppose $X$ is a compactum, $K$ is a component of $X$ and $V$ is a set open in $K$. Then there is a set $V^{\prime}$ open in $X$ such that $V^{\prime} \cap K=V$ and $\mathrm{Cl}\left(V^{\prime}\right) \cap K=\mathrm{Cl}(V)$.

Lemma 1.4. Suppose $X$ is a compactum with a snake-like continuum $K$ as a component and $C=\left(c_{1}, \cdots, c_{n}\right)$ is a chain covering $K$ where each $c_{i}$ is an open set in $K$. Then there is a chain $D=\left(d_{1}, \cdots, d_{n}\right)$ covering $K$ where each $d_{i}$ is an open set in $X$ and $d_{i} \cap K=c_{i}, i=1, \cdots, n$.

Proof. By the previous lemma we have that there is a set $d_{i}^{\prime}$ open in $X$ such that $d_{i}^{\prime} \cap K=c_{i}$ and $\mathrm{Cl}\left(d_{i}^{\prime}\right) \cap K=\mathrm{Cl}\left(c_{i}\right)$ for $i=1, \cdots, n$. Let 


$$
d_{i}=d_{i}^{\prime}-\bigcup\left\{\mathrm{Cl}\left(d_{j}^{\prime}\right) \mid j \in \varphi(i)\right\}
$$

where $\varphi(i)=\{j \mid j$ is an integer, $1 \leqq j \leqq n,|j-i|>1\}$. Then $D=$ $\left(d_{1}, \cdots, d_{n}\right)$ is a chain covering $K$ and $d_{i} \cap K=c_{i}, i=1, \cdots, n$.

Notation. If $C=\left\{C_{\alpha} \mid \alpha \in A\right\}$ and $D=\left\{D_{\beta} \mid \beta \in B\right\}$ are two collections of sets, $C \cap D=\left\{C_{\alpha} \cap D_{\beta} \mid \alpha \in A, \beta \in B, C_{\alpha} \cap D_{\beta} \neq \varnothing\right\}$. If, in addition, $E$ is a set $E \cap C=\left\{E \cap C_{\alpha} \mid \alpha \in A, E \cap C_{\alpha} \neq \varnothing\right\}$. Finally, $C^{*}=\bigcup\left\{C_{\alpha} \mid \alpha \in A\right\}$.

THEOREM 1.4. Suppose $X$ is a compactum each nondegenerate component of which is a snake-like continuum. Then $X$ is embeddable in $R^{2}$.

Proof. First we show that $X$ has arbitrarily small coverings whose nerves are embeddable in $R^{1}$. Let $U=\left(u_{1}, \cdots, u_{m}\right)$ be an arbitrary finite irreducible covering of $X$ and let $\left\{K_{\alpha} \mid \alpha \in A\right\}$ be the collection of components of $X$. Denote $\left\{u_{k} \mid u_{k} \in U, u_{k} \cap K_{\alpha} \neq \varnothing\right\}$ by $U_{\alpha}$. There exists a a chain $C_{\alpha}=\left(c_{1}^{\alpha}, \cdots, c_{n(\alpha)}^{\alpha}\right)$ covering $K_{\alpha}$ each of whose links is open in $K_{\alpha}$ and such that $C_{\alpha}>U_{\alpha}$ (i.e., $C_{\alpha}$ refines $U_{\alpha}$ ). By the previous lemma there is a chain $D_{\alpha}=\left(d_{1}^{\alpha}, \cdots, d_{n(\alpha)}^{\alpha}\right)$ covering $K_{\alpha}$ each of whose links is open in $X$ and such that $D_{\alpha} \cap K_{\alpha}=C_{\alpha}$.

Now letting $v_{j}^{\alpha}=\bigcap\left\{u_{k} \mid u_{k} \in U_{\alpha}, c_{j}^{\alpha} \subset u_{k}\right\}$ we define $E_{\alpha}$ to be $\left\{d_{j}^{\alpha} \cap v_{j}^{\alpha} \mid j=1, \cdots, n(\alpha)\right\}$. Then $E_{\alpha}$ is a chain covering $K_{\alpha}$ whose links are open in $X$ and such that $E_{\alpha}>U_{\alpha}$. Finally we define

$$
E_{\alpha}^{\prime}=\left\{d_{j}^{\alpha} \cap v_{j}^{\alpha}-\bigcup\left\{K_{\beta} \mid \beta \in A, K_{\beta} \not \subset E_{\alpha}^{*}\right\} \mid d_{j}^{\alpha} \cap v_{j}^{\alpha} \in E_{\alpha}\right\} .
$$

Then $E_{\alpha}^{\prime}$ is a chain covering $K_{\alpha}$ whose links are open in $X$ and such that $E_{\alpha}^{\prime}>U_{\alpha}$. We can partition $X$ as follows. There is a collection $P=\left(P_{1}, \cdots, P_{n}\right)$ of disjoint open and closed sets whose union is $X$. Then $\left\{P \cap E_{\alpha}^{\prime} \mid \alpha \in A\right\}$ is a covering of $X$ and since the latter is compact we need only elements from a finite number of the chains $\left\{P \cap E_{\alpha}^{\prime} \mid \alpha \in A\right\}$ to cover $X$, say $Q=\left(Q_{1}, \cdots, Q_{s}\right)$ where $Q_{j}=P_{j} \cap E_{\alpha}^{\prime}$ for some $j, 1 \leqq$ $j \leqq n$ and $\alpha \in A$. Then $Q$ is a covering of $X, Q>U$ and each nondegenerate component of the nerve of $Q$ is an arc. Hence the nerve of $Q$ is embeddable in $R^{1}$ and since there is a $Q$-mapping of $X$ into the nerve of $Q$ we have that $X$ is the inverse limit of an inverse sequence each coordinate space of which is contained in $R^{1}$ (see [9, Lemma 1.6]). Thus by Theorem 1 of [13] $X$ is embeddable in $R^{2}$.

THEOREM 1.5. ([8]) If $X$ is a planar compactum each nondegenerate component of which is a snake-like continuum (pseudo-arc), then $X$ is embeddable in a snake-like continuum (pseudo-arc). 
THEOREM 1.6. If $X$ is a compactum, then the following are equivalent:

(a) every nondegenerate component of $X$ is a snake-like continuum.

(b) $d X \leqq d Y$ for some snake-like continuum $Y$ (i.e., $X$ is embeddable in $Y$ ).

(c) $q X \leqq q I$.

Proof. First, (a) implies (b) by Theorems 1.4 and 1.5. Second, (b) implies (c) since $q X \leqq q Y \leqq q I$. Third, (c) implies (a) since, for any nondegenerate component $K$ of $X, q K \leqq q I$ so that $K$ is a snakelike continuum by Lemma 1.2 .

THEOREM 1.7. If $X$ is a compactum, then the following are equivalent:

(a) every nondegenerate component of $X$ is a pseudo-arc.

(b) $d X \leqq d \psi$.

(c) $q X \leqq q \psi$.

Proof. First, (a) implies (b) by Theorems 1.4 and 1.5. Second, it is obvious that (b) implies (c). Third, (c) implies (a) since, for any nondegenerate component $K$ of $X, q K \leqq q \psi$ so that $K$ is a pseudo-arc by Lemma 1.1 and 1.2.

THEOREM 1.8. In the class of metric spaces the Cantor discontinuum $C$ immediately precedes the pseudo-arc $\psi($ i.e., $q C \ll q \psi)$.

Proof. Suppose not, i.e., $q C<q X<q \psi$ for some metric space $X$. Then $X$ is compact and not totally disconnected (otherwise $q X \leqq q C)$. Therefore $X$ contains a nondegenerate continuum and so $q X=q \psi$ by Theorem 1.1. But this is a contradiction.

\section{Quasi dimension type of linear graphs.}

Definition 2.1. By a linear graph we mean a finite connected 1-dimensional geometric complex. A tree is an acyclic linear graph. An end point of a linear graph is a vertex which is contained in only one edge (1-simplex). A branch point is a vertex which is contained in more than two edges. A tree which is homeomorphic to the cone over $n(\geqq 2)$ points is called an $n$-od. A dendrite is a locally connected continuum which contains no simple closed curve. In a tree the unique arc with end points $p, q$ is denoted by $[p q] ;[p q]=[p q]-p$, etc.

Definition 2.2. For a positive integer $n$, we say the curve $X$ 
is of order $\leqq n$ at the point $p$ (in symbols: $\operatorname{ord}_{p} X \leqq n$ ) if, for every $\varepsilon>0$, there exists an open set $U$ of $X$ such that $p \in U, \delta(U)<\varepsilon$ and cardinality of the boundary of $U \leqq n$. Furthermore, if $\operatorname{ord}_{p} X \leqq n$ and it is false that $\operatorname{ord}_{p} X \leqq m$ for any $m<n$, then we say $\operatorname{ord}_{p} X=n$. We also define for a linear graph $X$ the following

$$
\sigma(X)=2+\sum\left(\operatorname{ord}_{p} X-2\right)
$$

and

$$
\beta(X)=\sum \operatorname{ord}_{p} X
$$

the summations being extended over all branch points of $X$.

LEMMA 2.1. If $X$ is a linear graph, then $\beta(X)-\sigma(X)=2(m-1)$ where $m$ is the number of branch points of $X$.

Lemma 2.2. ([14, Th. 5.5]) If $X$ is a linear graph, then $\operatorname{dim} C(X)=\sigma(X)$. Moreover, $C(X)$ is a polyhedron.

THEOREM 2.1. If $X$ and $Y$ are linear graphs and, for every $\varepsilon>0$, there is an $\varepsilon$-map $f_{\varepsilon}: X \rightarrow Y$, then for $\varepsilon$ sufficiently small

$$
\sigma(X) \leqq \sigma\left(f_{\mathrm{c}}[X]\right) \text {. }
$$

Proof. For any $\varepsilon>0$ we have $f_{\varepsilon}[X]$ is subcontinuum of $Y$ and hence is also a linear graph. The map $f_{\varepsilon}^{\prime}$ induced by $f_{\varepsilon}$ (see Theorem 1.3) is an $\varepsilon$-mapping of $C(X)$ into $C\left(f_{\varepsilon}[X]\right)$. Therefore, for $\varepsilon$ sufficiently small we have by Alexandroff's Theorem on the approximation to compact spaces by polyhedra (see [12, p. 72]) that

$$
\sigma(X)=\operatorname{dim} C(X) \leqq \operatorname{dim} C\left(f_{\varepsilon}[X]\right)=\sigma\left(f_{\varepsilon}[X]\right) .
$$

Corollary 2.1. If $X$ and $Y$ are linear graphs and $q X \leqq q Y$, then $\sigma X \leqq \sigma Y$.

CoROLlaRY 2.2. If $X$ and $Y$ are linear graphs and $q X \leqq q Y$, then $\beta(X) \leqq \beta(Y)$.

Notation. For any metric space $X$ and $\varepsilon>0$ we define

$$
V(x, \varepsilon)=\{y \in X \mid \rho(x, y) \leqq \varepsilon\} .
$$

Theorem 2.2. If $X$ and $Y$ are linear graphs, then the following statements are equivalent.

(a) $q X=q Y$. 
(b) $d X=d Y$.

(c) $X$ and $Y$ are homeomorphic.

Proof. The implications $(\mathrm{c}) \rightarrow(\mathrm{b}) \rightarrow($ a) are trivial. Now assume $q X=q Y$. Denote the branch points of $X$ by $p_{1}, \cdots, p_{m}$ and the branch points of $Y$ by $q_{1}, \cdots, q_{n}$. We consider $X$ and $Y$ to have the decompositions determined by taking the branch points and end points as the vertices. Let $\nu=1 / 2 \min \left\{\rho\left(v_{i}, v_{j}\right) \mid v_{i}, v_{j}\right.$ are distinct vertices of $Y$ \}. First we show that $m=n$. There is a $\Delta_{i}, 0<\Delta_{i}<\nu / 2$, such that, for any positive number $\Delta \leqq \Delta_{i}$,

$$
\operatorname{ord}_{q_{i}} V\left(q_{i}, \Delta\right)=\operatorname{ord}_{q_{i}} Y, \quad \text { for } i=1, \cdots, n .
$$

This in turn implies that $\sigma\left(V\left(q_{i}, \Delta\right)\right)=\operatorname{ord}_{q_{i}} Y$. Let $V_{i}=V\left(q_{i}, \Delta_{i}\right)$ and $\sigma_{i}=\sigma\left(V_{i}\right)$. By hypothesis for every $\varepsilon>0$ there is an $\varepsilon$-map $g_{\varepsilon}: Y \rightarrow X$. So

$$
2<\operatorname{ord}_{q_{i}} V_{i}=\sigma_{i} \leqq \sigma\left(g_{\eta_{1}}\left[V_{i}\right]\right)
$$

where $\eta_{1}=\min \left\{\varepsilon_{1}, \cdots, \varepsilon_{n}\right\}$ and $\varepsilon_{i}$ is sufficiently small so that $\sigma\left(V_{i}\right) \leqq$ $\sigma\left(g_{\varepsilon_{i}}\left[V_{i}\right]\right)$ for $i=1, \cdots, n$. Now since $V_{i}$ is a $\sigma_{i}$-od we have

$$
V_{i}=\bigcup_{r=1}^{\sigma_{i}}\left[q_{i} y_{i}^{r}\right]
$$

where the $\left(q_{i} y_{i}^{r}\right]$ are disjoint arcs.

We now define $Y^{\prime}$ to be the subdivision of $Y$ induced by taking as the vertices of $Y^{\prime}$ those of $Y$ and all the points $y_{i}^{r}, i=1, \cdots, n$; $r=1, \cdots, \sigma_{i}$. (Note that $Y$ and $Y^{\prime}$ are homeomorphic and have the same branch points and end points.) Let the end points $Y^{\prime}$ be denoted by $e_{k}^{r}$ where the subscript and superscript correspond to those of the $y_{k}^{r}$ which is adjacent to $e_{k}^{r}$, (i.e., $\left[e_{k}^{r} y_{k}^{r}\right]$ is an edge of $Y^{\prime}$ ). Then note that $Y^{\prime}-\bigcup_{i=1}^{n} V_{i}$ is the union of disjoint arcs of two kinds:

(1) $\left[e_{k}^{r} y_{k}^{r}\right)$ for every end point $e_{k}^{r}$ of $Y^{\prime}$, and

(2) $\left(y_{k}^{r} y_{m}^{s}\right)$ where $\left[y_{k}^{r} y_{m}^{s}\right]$ is an edge of $Y^{\prime}$ and $y_{k}^{r}$ and $y_{m}^{s}$ are end points of $V_{k}$ and $V_{m}$, respectively.

Let

$$
\eta=\min \left\{\eta_{1}, \lambda_{1}, \cdots, \lambda_{n}\right\}
$$

where

$$
\begin{gathered}
\lambda_{i}=\min \left[\min \left\{\rho\left(V_{i},\left[e_{k}^{r} y_{k}^{r}\right]\right) \mid\left[e_{k}^{r} y_{k}^{r}\right] \cap V_{t}=\varnothing \text { for any } t \neq k\right\},\right. \\
\min \left\{\rho\left(V_{i},\left[y_{k}^{r} y_{m}^{s}\right]\right) \mid\left[y_{k}^{r} y_{m}^{s}\right] \text { is an edge of } Y^{\prime}\right. \text { and } \\
\left.\left.\left[y_{k}^{r} y_{m}^{s}\right] \cap V_{t}=\varnothing \text { for any } t \neq k \text { or } m\right\}\right] .
\end{gathered}
$$

Now for any $\eta$-map, $g_{\eta}$, we have that the sets $g_{\eta}\left[V_{i}\right], \cdots, g_{\eta}\left[V_{n}\right]$ are 
disjoint (since $\left.\rho\left(V_{i}, V_{j}\right) \geqq \nu>\eta\right)$. Since $\sigma\left(g_{\eta}\left[V_{i}\right]\right)>2$ we have that $g_{\eta}\left[V_{i}\right]$ must contain at least one branch point of $X$ for $i=1, \cdots, n$. Therefore $n \leqq m$ and by a symmetric argument $m \leqq n$ and so $m=n$. Moreover, $g_{\eta}\left[V_{i}\right]$ contains exactly one branch point of $X$, say $p_{j(i)}$. Likewise $f_{\gamma}\left[W_{i}\right]$ contains exactly one branch point of $Y^{\prime}$ say $q_{k(i)}$ (where $W_{i}=V\left(p_{i}, \delta_{i}\right)$, with $\delta_{i}$ chosen like $\Delta_{i}, \gamma$ chosen like $\eta$ and $X^{\prime}$ determined like $\left.Y^{\prime}\right)$. So $\operatorname{ord}_{p_{j(i)}} X \geqq \sigma\left(g_{\eta}\left[V_{i}\right]\right) \geqq \sigma_{i}$ and $\operatorname{ord}_{q_{k(i)}} Y^{\prime} \geqq$ $\sigma\left(f_{r}\left[W_{i}\right]\right) \geqq \sigma\left[W_{i}\right]$. Therefore,

$$
\begin{aligned}
\sigma\left(X^{\prime}\right) & =2+\sum_{i=1}^{m}\left(\operatorname{ord}_{p_{j(i)}} X^{\prime}-2\right) \geqq 2+\sum_{i=1}^{m}\left(\sigma_{i}-2\right) \\
& =2+\sum_{i=1}^{m}\left(\operatorname{ord}_{q_{i}} Y^{\prime}-2\right)=\sigma\left(Y^{\prime}\right)
\end{aligned}
$$

and since $\operatorname{ord}_{p_{j(i)}} X^{\prime} \geqq \sigma_{i}$ for each $i$ we have $\operatorname{ord}_{p_{j(i)}} X^{\prime}=\operatorname{ord}_{q_{i}} Y^{\prime}$ for $i=1, \cdots, m$. Likewise $\operatorname{ord}_{q_{k(i)}} Y^{\prime}=\operatorname{ord}_{p_{i}} X^{\prime}$ for $i=1, \cdots, m$.

We can now write $g_{\eta}\left[V_{i}\right]$ as follows: $g_{\eta}\left[V_{i}\right]=\bigcup_{r=1}^{o_{i}}\left[p_{j(i)} x_{j(i)}^{r}\right]$ and the $\left(p_{j(i)} x_{j(i)}^{r}\right]$ are disjoint arcs. Thus relying on the way in which $\eta$ was chosen we have $X^{\prime}-\bigcup_{i=1}^{n} g_{\eta}\left[V_{i}\right]$ is the union of disjoint arcs of two kinds:

(3) $\left[d_{j(k)}^{r} x_{j(k)}^{r}\right)$ for every end point $d_{j(k)}^{r}$ of $X^{\prime}$ which is in $X^{\prime}-\bigcup_{i=1}^{n} g_{\eta}\left[V_{i}\right]$ (the subscript and superscript of $d$ being determined by the fact that $\left[d_{j(k)}^{r} x_{j(k)}^{r}\right)$ does not contain and end point of any $g_{\eta}\left[V_{i}\right]$, for $i=1, \cdots, n$, and

(4) $\left(x_{j(k)}^{r} x_{j(m)}^{s}\right)$ for end points $x_{j(k)}^{r}$ and $x_{j(m)}^{s}$ of $g_{\eta}\left[V_{k}\right]$ and $g_{\eta}\left[V_{m}\right]$, respectively, and where $g_{\eta}\left(y_{i}^{r}\right) \in\left(p_{j(i)} x_{j(i)}^{r}\right], i=1, \cdots, n$.

We now define a homeomorphism $h$ of $Y^{\prime}$ onto $X^{\prime}$ as follows: and

(5) $\left(h \mid V_{i}\right): V_{i} \rightarrow g_{\eta}\left[V_{i}\right]$ is a homeomorphism such that $h\left(q_{i}\right)=p_{j(i)}$

$$
h\left(y_{i}^{r}\right)=\left\{\begin{array}{l}
z_{j(i)}^{r}\left(=\text { midpoint of }\left[p_{j(i)} x_{j(i)}^{r}\right]\right) ; \text { if } d_{j(i)}^{r} \in g_{\eta}\left[V_{i}\right] \\
x_{j(i)}^{r}, \text { otherwise }
\end{array}\right\}
$$

where $g_{\eta}\left(y_{i}^{r}\right) \in\left(p_{j(i)} x_{j(i)}^{r}\right], i=1, \cdots, n$, and $r=1, \cdots, \sigma_{i}$.

(6) $\left(h \mid\left[y_{k}^{r} y_{m}^{s}\right]\right):\left[y_{k}^{r} y_{m}^{s}\right] \rightarrow\left[x_{j(k)}^{r} x_{j(m)}^{s}\right]$ is a homeomorphism such that $h\left(y_{k}^{r}\right)=x_{j(k)}^{r}$ and $h\left(y_{m}^{s}\right)=x_{j(m)}^{s}$, for any edge $\left[y_{k}^{r} y_{m}^{s}\right]$ in $Y^{\prime}$ whose end points are also end points of $V_{k}$ and $V_{m}$, respectively, and $k \neq m$.

(7) $\left(h \mid\left[e_{i}^{r} y_{i}^{r}\right]\right):\left[e_{i}^{r} y_{i}^{r}\right] \rightarrow\left[d_{j(i)}^{r} h\left(y_{i}^{r}\right)\right]$ is a homeomorphism such that $h\left(e_{i}^{r}\right)=d_{j(i)}^{r} ; h\left(y_{i}^{r}\right)$ is as in (5).

This determines a homeomorphism $h$ of $Y^{\prime}$ onto $X^{\prime}$ and so $Y$ and $X$ are homeomorphic. Therefore we have (a) $\rightarrow$ (c).

The first of following examples shows that $q X \leqq q Y$ does not imply $d X \leqq d Y$ even in the case that $X$ and $Y$ are trees. The second shows that Theorem 2.2 is not true for dendrites. 
ExAmple 2.1. Let $X$ be a 4 -od and $Y$ the space obtained by taking the union of two vertical segments with end points $(1 / 4,0)$ and $(3 / 4,0)$ and the closed unit interval $[0,1]$. Then $q X \leqq q Y$ since an arc of arbitrarily small diameter with the branch point of $X$ as one end point can be projected onto an arc of the same kind on another branch of $X$ thereby obtaining a space homeomorphic to $Y$. But obviously neither $X$ nor $Y$ is embeddable in the other.

EXAMPLE 2.2. Let $X$ be the union of the sets (1), (2) and (3) and let $Y$ be the union of the sets (1) and (2) where

(1) the closed unit interval $[0,1]$,

(2) the vertical segments with end points $(1 / n, 0)$ and $(1 / n, 1 / n)$, $n=2,3, \cdots$,

(3) the segment given by $y=(1 / 2)-x, 3 / 8 \leqq x \leqq 1 / 2$. Then $q X \leqq q Y$ (see the previous example) and $q Y \leqq q X$ since $Y$ is a closed subset of $X$. So $q X=q Y$ but $d X \not d Y$.

\section{Quasi dimension type of Peano curves.}

Definition 3.1. A curve is a 1-dimensional continuum and a Peano curve (continuum) is a locally connected curve (continuum). A curve $X$ is essentially nonplanar, if there is an $\varepsilon>0$ such that $X$ cannot be $\varepsilon$-mapped onto any planar curve. If a space is the unit sphere of $R^{n+1}$, then it is called a $n$-sphere and is denoted by $S^{n}$. A space homeomorphic to $S^{1}\left(S^{2}\right)$ is called a simple closed curve (spherical surface).

Definition 3.2. Kuratowski [15] described four Peano curves $K_{1}$ (=1-skeleton of a tetrahedron with midpoints of a pair of nonadjacent edges joined by a segment), $K_{2}$ (= complete graph on 5 vertices), $K_{3}$ and $K_{4}$ which are indicated in Figures 1, 2, 3, and 4, respectively. A topological image of anyone of the four curves $K_{1}$, $K_{2}, K_{3}, K_{4}$ is called a skew curve. A topological image of $K_{1}$ or $K_{2}$ $\left(K_{3}\right.$ or $\left.K_{4}\right)$ is called a primitive (secondary) skew curve.

Kuratowski showed that every nonplanar linear graph contains a primitive skew curve. Claytor [7] managed to use the secondary skew curves to obtain the following elegant result: A Peano continuum which is not homeomorphic with a subset of $S^{2}$ necessarily contains a topological image of a skew curve. As a special case he also proved: Every cyclic Peano continuum which is not homeomorphic with a subset of $S^{2}$ contains a topological image of a primitive skew curve.

Definition 3.3. The Sierpinski (or universal plane) curve, $\mathfrak{\subseteq}$, and the Menger (or universal) curve, $\mathfrak{M}$, are different 1-dimensional locally 


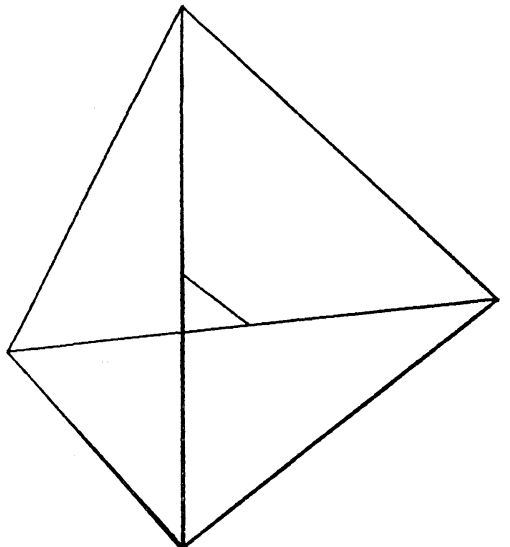

FIG. 1

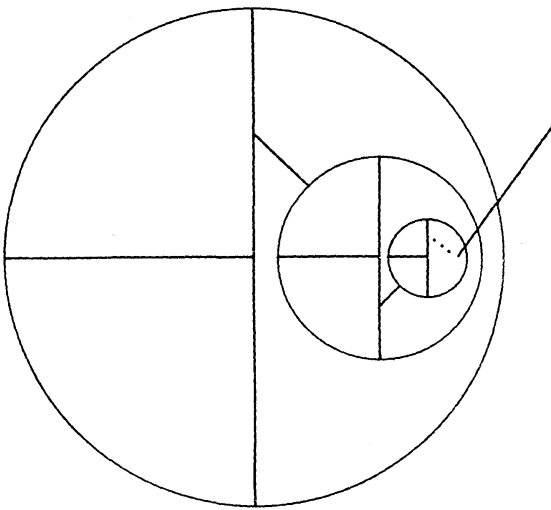

FIG. 3

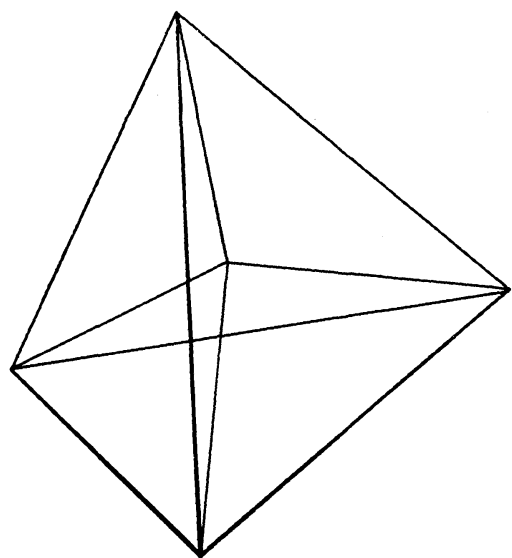

FIG. 2

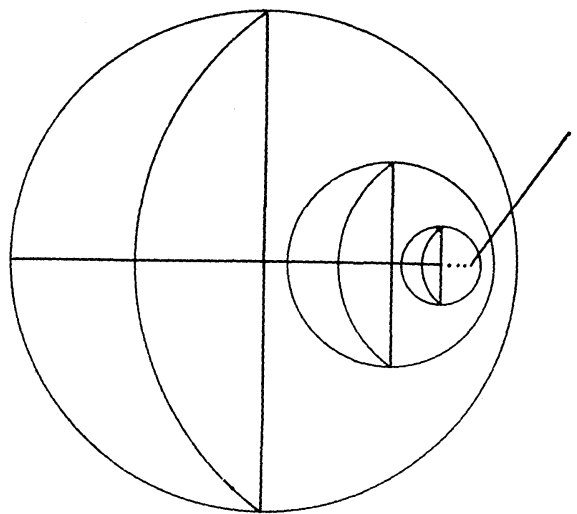

FIG. 4

connected analogues of the Cantor discontinuum. The Sierpinski curve can be obtained by considering a square and by successively deleting first the open middle-ninth of that square, second the middle-ninths of each of the eight squares remaining, third the middle-ninths of each of the 64 squares remaining, etc. The Menger curve may be defined as the set of all points of the unit cube which project in each of the three directions (of the edges) into Sierpinski curves constructed as above on the various faces of the cube. The Menger (Sierpinski) curve contains homeomorphic images of all 1-dimensional (planar) continua. Actually a more general statement is true, i.e., any 1dimensional separable metric space can be embedded in $\mathfrak{M}$ and any 1-dimensional planar set can be embedded in $\subseteq$ (see [20, p. 106]). So for any subset $X$ of $R^{3}\left(R^{2}\right)$ we have that $q X \leqq q \mathfrak{M}(q \subseteq)$ implies $d X \leqq d \mathfrak{M}(d \subseteq)$.

Mazurkiewicz [18] has shown the following.

THEOREM 3.1. An essentially nonplanar Peano curve contains a 
primitive skew curve.

Corollary. If $X$ is a Peano curve which does not contain a primitive skew curve, then $q X \leqq q \subseteq$.

THEOREM 3.2. [11] If $X$ is a compact polyhedron of dimension $n \neq 2$ such that $q X \leqq q R^{2 n}$, then $d X \leqq d R^{2 n}$.

Combining these two results we have the next theorem.

THEOREM 3.3. If $X$ is a Peano curve, then $q X \leqq q R^{2}$ if and only if $X$ does not contain a primitive skew curve.

Proof. Suppose $X$ contains a primitive skew curve, $K$, and suppose $q X \leqq q R^{2}$, then $q K \leqq q X \leqq q R^{2}$. Therefore, by the previous theorem $d K \leqq d R^{2}$, but this is impossible. The other half of the theorem is just a restatement of Theorem 3.1.

Claytor's fundamental result [7] in this area is stated next.

THEOREM 3.4. A Peano continuum $X$ is embeddable in $S^{2}$ if and only if $X$ does not contain a skew curve.

Corollary. A Peano continuum $X$ which is not a spherical surface is planar if and only if $X$ does not contain a skew curve.

The next two theorems show how quasi dimension type is related to the above results in a particular setting.

Theorem 3.5. If $X$ is a Peano curve which does not contain a secondary skew curve, then the following are equivalent.

(a) $d X \leqq d R^{2}$.

(b) $q X \leqq q \subseteq$.

(c) $q X \leqq q R^{2}$.

(d) $X$ does not contain a primitive skew curve.

Proof. First, (a) implies (b) since if $X$ is planar it is embeddable in $\subseteq$. Second, (b) implies (c) since $q \subseteq \subseteq q R^{2}$. Third, (c) implies (d) is just half of Theorem 3.3. Fourth, (d) implies (a) follows from Theorem 3.4.

THEOREM 3.6. If $X$ is a Peano continuum which does not contain a secondary skew curve, then the following are equivalent.

(a) $d X \leqq d R^{2}$.

(b) $q X \leqq q R^{2}$. 
(c) $X$ is not a spherical surface and does not contain a primitive skew curve.

Proof. First, (a) implies (b) is trivial. Second, (b) implies (c) follows from Theorem 3.3 and the Borsuk-Ulam Theorem, i.e., for any mapping of $f: S^{n} \rightarrow R^{n}$, there is a pair of antipodal points of $S^{n}$ with the same image under $f$ (so that $q S^{2} \geqq q R^{2}$ ). Finally (c) implies (a) as a result of Theorem 3.4.

REMARK 3.1. Note that $K_{3}$ and $K_{4}$ are such that $q K_{3} \leqq q R^{2}$ and $q K_{4} \leqq q R^{2}$. To see that the former is true choose any $\varepsilon>0$. Then take a circle $S$ in the defining sequence of $K_{3}$ of diameter $<\varepsilon / 3$. Then shrinking $S$ and its interior (in $R^{2}$ ) to a point clearly yields a space embeddable in $R^{2}$. The same technique works for showing that $q K_{4} \leqq q R^{2}$. This shows that necessity of requiring that $X$ contain no secondary skew curve in Theorem 3.5 and 3.6. Since the dyadic solenoid $D$ is such that $q D \leqq q S^{1}<q R^{2}$ but $d D \leqq d R^{2}$, the need for requiring $X$ to be locally connected in the previous two theorems is evinced.

Mazurkiewicz [18] also pointed out that the following two theorems are consequences of Theorem 3.1.

THEOREM 3.7. If $X$ is a curve and $q K_{1} \leqq q K$, then $d K_{1} \leqq d X$.

THEOREM 3.8. If $X$ is a curve and $q K_{2} \leqq q X$, then $d K_{1} \leqq d X$ or $d K_{2} \leqq d X$.

As a result of Theorems 3.7 and 2.1 we have the following.

THEOREM 3.9. The primitive skew curves, $K_{1}$ and $K_{2}$, are not comparable with respect to quasi dimension type.

THEOREM 3.10. The secondary skew curves, $K_{3}$ and $K_{4}$ are not comparable with respect to quasi dimension type.

Proof. Suppose $q K_{3} \leqq q K_{4}$. Let $A_{1}$ be a subset of $K_{3}$ which is a circumscribed figure $T$ (i.e., $A_{1}=S \cup B$ where $S$ is a simple closed curve, $B$ is a 3-od with center $x$ and end points $a_{1}, a_{2}$, and $a_{3}$; and $\left.S \cap B=a_{1} \cup a_{2} \cup a_{3}\right)$. Let $A=A_{1} \cup[y b] \cup[z c]$ where $[y b]$ and $[z c]$ are arcs, $[y b] \cap A_{1}=y \in\left(x a_{1}\right)$ (an open arc contained in $B$ ), and $[z c] \cap A_{1}=z$ (an element of the open arc of $S$ from $a_{1}$ to $a_{2}$ which does not contain $\left.a_{3}\right)$. Then, for $\varepsilon$ sufficiently small, the $\varepsilon$-image $A$ contains a curve homeomorphic to $A$. However, $K_{4}$ contains no such curve so we have a contradiction. 
Suppose $q K_{4} \leqq q K_{3}$. Let $A$ denote a circumscribed figure $X$ (i.e., $A=S \cup B$ where $S$ is a simple closed curve, $B$ is a 4 -od with center $x$ and end points $a_{1}, a_{2}, a_{3}$ and $a_{4}$; and $\left.S \cap B=a_{1} \cup a_{2} \cup a_{3} \cup a_{4}\right)$. Then, for $\varepsilon$ sufficiently small, the $\varepsilon$-mage of $A$ contains either a curve which is homeomorphic to $A$, or a circumscribed figure $H$. However, $K_{3}$ contains no such curves so we have a contradiction.

Corollary. No two of the skew curves are comparable with respect to quasi dimension type.

4. Quasi dimension type of curves. Recall the following notation: $I=$ closed unit interval, $D=$ dyadic solenoid, $\psi=$ pseudo-arc, $\mathfrak{S}=$ Sierpinski curve and $\mathfrak{M}=$ Menger curve. Then from the results of $\S 1$ and $\S 2$ we have the following.

THEOREM 4.1. If $X$ is a snake-like continuum, then

(1) $q \psi \leqq q X \leqq q I$;

(2) $X$ contains an arc if and only if $q I \leqq q X$;

(3) $X$ is a pseudo-arc if and only if $q X \leqq q \psi$.

Definition 4.1. A circular chain is a chain subjected to the added condition that its first and last links intersect. A continuum $X$ is called circle-like if for each $\varepsilon>0, X$ can be covered with a circular chain of mesh $<\varepsilon$.

THEOREM 4.2. If $X$ is a circle-like continuum, then

(1) $q \psi \leqq q X \leqq q S^{1}$

(2) $X$ is a simple closed curve if and only if $q S^{1} \leqq q X$;

(3) $X$ is a pseudo-arc if and only if $q X \leqq q \psi$.

(4) $q X \leqq q D$ implies that $q X \leqq q I$ or $X$ is not planar.

Proof. The first inequality of (1) follows from the fact that any circle-like continuum contains a snake continuum and Theorem 1.2'. The second inequality of (1) follows from the fact that a continuum has $\varepsilon$-mappings into the nerves of its coverings.

Only one implication of (2) is nontrivial. Assume $q S^{1} \leqq q X$. This implies that $X$ contains a simple closed curve $S$ and since $q X \leqq q S^{1}$ there are $\varepsilon$-mappings, $f_{\varepsilon}$, of $X$ into $S^{1}$. Then, for $\varepsilon$ sufficiently small, $f_{\varepsilon}$ must take $S$ onto all of $S^{1}$. Therefore $S=X$. Condition (4) follows from the fact that $D$ is not the continuous image of any planar continuum (see [10]).

DEFINITION 4.2. A tree chain is a finite collection of open sets whose nerve is a tree. A continuum $X$ is called tree-like if for each 
$\varepsilon>0, X$ can be covered with a tree chain of mesh $>\varepsilon$.

Definition 4.3. For any positive integer $n, a$ curve $X$ is said to be of order less than or equal to $n$ (in symbols: ord $X \leqq n$ ), if $\operatorname{ord}_{p} X \leqq n$ for each $p \in X$ (see Definition 2.2). Furthermore, if ord $X \leqq n$ and it is false that ord $X \leqq m$ for any $m<n$ (i.e., $\operatorname{ord}_{p} X=n$ for some $p \in X)$, then ord $X=n$.

DeFINITION 4.4. For any positive integer $n$, a continuum $X$ is said to be of $\tau$-order less than or equal to $n$ (in symbols: $\tau$-ord $X \leqq n$ ), if $X$ has coverings of arbitrarily small whose nerves are trees of order $\leqq n$. (The usual convention prevails in defining $\tau$-ord $X=n$.) If we replace " $\tau$ " by " $\gamma$ " and "trees" by "linear graphs", then we obtain the definition of $\gamma$-ord $X \leqq n$.

Menger [19, p. 318] has shown that there is a dendrite $\mathfrak{U}$ (in the plane) which is universal for dendrites (i.e., any dendrite can be embedded in $\mathfrak{u}$ ). Moreover, he showed that for any positive integer $n \geqq 2$ there is a dendrite $\mathfrak{U}_{n}$ of order $n$ which is universal for all dendrites of order $n$.

Lemma 4.1. If $X$ is a tree, then $\tau$-ord $X \leqq 3$.

Proof. Let $b_{1}, \cdots, b_{n}$ denote the branch points of $X, k_{i}=\operatorname{ord}_{b_{i}} X$ and $\nu=1 / 2 \min \left\{\rho\left(v_{i}, v_{j}\right) \mid v_{i}, v_{j}\right.$ are distinct vertices of $\left.X\right\}$. There is a $\Delta_{i}, 0<\Delta_{i}<\nu / 2$, such that, for any positive number $\Delta \leqq \Delta_{i}$, $\operatorname{ord}_{b_{i}} V\left(b_{i}, \Delta\right)=k_{i}$, for $i=1, \cdots, n$. Let $r=(1 / 2) \min \left\{\Delta_{i} \mid i=1, \cdots, n\right\}$. We will replace each $k_{i}$-od in $X$ by a $\left(k_{i}-2\right)$-comb (a $m$-comb is a tree homeomorphic to the union of the closed interval $[0, m+1]$ and $m$ vertical segments from $(i, 0)$ to $(i, 1)$ for $i=1, \cdots, m)$. This will yield a new tree $Y$ of order $\leqq 3$ which is the $\varepsilon$-image of $X$ for each $\varepsilon>0$.

For a fixed branch point $b_{i}{ }^{\prime}$ consider the $r$-neighborhood $S\left(b_{i}, r\right)$ and its boundary points $y_{1}, \cdots, y_{k_{i}}$. Let $A_{1}$ be an are which intersects $X$ only in its end points $y_{1}$ and $y_{2}$. Let $z_{3}, \cdots, z_{k_{i}}$ be $\left(k_{i}-2\right)$ distinct points of $A_{1}-\left(y_{1} \cup y_{2}\right)$. Now, for $3 \leqq j \leqq k_{i}$, let $A_{i-1}$ be an arc which intersects $X \cup A_{1} \cup \cdots \cup A_{j-2}$ only in its end points $z_{j}$ and $y_{j}$. Finally let $B_{i}=\bigcup\left\{A_{j} \mid j=1, \cdots, k_{i}-1\right\}$ (so that $B_{i}$ is a $\left(k_{i}-2\right)$-comb) and let $Y=\left[X-\bigcup_{i=1}^{n} S\left(b_{i}, r\right)\right] \cup\left[\bigcup_{i=1}^{n} B_{i}\right]$. Using the techniques of Theorem 2.2 and Example 2.1 it is not difficult to see that $X$ can be $\varepsilon$-mapped onto $Y$ for all $\varepsilon>0$.

Now let $V$ be a covering of $X$ with Lebesque number $\eta$. There exists a subdivision of $Y$ fine enough so that, for the associated 
covering. $U$ by stars of vertices, there exists an $\varepsilon$-mapping $f$ of $X$ onto $Y$ with the property that the mesh $f^{-1}(U)<\eta$. Then $f^{-1}(U)$ refines $V$ and its nerve is homeomorphic to $Y$. Hence $X$ has coverings of arbitrarily small mesh whose nerves are trees of order $\leqq 3$, i.e., $\tau$-ord $X<3$.

THEOREM 4.3. A continuum $X$ is tree-like if and only if $\tau$-ord $X \leqq 3$.

Proof. Suppose $X$ is tree-like (the other implication follows immediately from the definition of $\tau$-order). Then $X$ has coverings $\left\{V_{i}\right\}$ whose nerves $N\left(V_{i}\right)=N_{i}$ are trees and such that mesh $V_{i} \leqq 1 / i$. Moreover, for any $\varepsilon>0$, there is an $\varepsilon$-mapping $f_{\varepsilon}$ of $X$ into some $N_{i}$. Now by the previous lemma each $N_{i}$ has coverings $\left\{W_{i}^{j}\right\}$ whose nerves are trees of order $\leqq 3$ and such that mesh $W_{i}^{j} \leqq 1 / j$.

In view of the compactness of $X$, for any $\varepsilon>0$, there is a positive integer $i$ and a $\eta>0$ such that: if $A \subset N_{i}$ and $\delta(A)<\eta$, then $\delta\left(f_{\varepsilon}^{-1}(A)\right)<\varepsilon$. So for $j$ large enough so that mesh $W_{i}^{\jmath}<\eta$ we have that mesh $f_{\varepsilon}^{-1}\left(W_{i}^{j}\right)<\varepsilon$. Moreover, $N\left(f_{\varepsilon}^{-1}\left(W_{\imath}^{j}\right)\right)$ is a tree of order $\leqq 3$ and therefore $\tau$-ord $X \leqq 3$.

Lemma 4.2. If $X$ and $Y$ are continua, $Y$ is tree-like and $q X \leqq q Y$, then $X$ is tree-like.

THEOREM 4.4. A continuum $X$ is tree-like if and only if $q X \leqq$ $q \mathfrak{U}_{3}$.

Proof. Suppose $X$ is tree-like. Then by the previous theorem $Y$ has coverings of arbitrarily small mesh whose nerves are trees of $\tau$-order $\leqq 3$. So these nerves are embeddable in $\mathfrak{U}_{3}$. Therefore, for any $\varepsilon>0, X$ can be $\varepsilon$-mapped into $\mathfrak{H}_{3}$, i.e., $q X \leqq q \mathfrak{U}_{3}$. Conversely, suppose $q X \leqq q \mathfrak{u}_{3}$. Now $\mathfrak{U}_{3}$ is a dendrite and dendrites (being planar and not containing a subcontinuum which separates the plane) are tree-like (see [5, p. 656]). Thus we have by Lemma 4.2 that $X$ is tree-like.

Corollary. For every positive integer $i, q \mathfrak{U}_{3}=q \mathfrak{U}_{3+i}=q \mathfrak{U}$.

Proof. Since $\mathfrak{U}_{i} \subset \mathfrak{U}_{i+1} \subset \mathfrak{U}$, we have that $q \mathfrak{U}_{i} \leqq q \mathfrak{U}_{i+1} \leqq q \mathfrak{U}$ for each positive integer $i$. Since $\mathfrak{U}_{\imath}$ and $\mathfrak{u}$ are dendrites they are also tree-like. Thus by the previous theorem $q \mathfrak{U}_{i}$ and $q \mathfrak{U} \leqq q \mathfrak{U}_{3}$ and so the desired result follows.

Since there are tree-like continua which fail to contain a snakelike subcontinuum we have as an open question the following. 
Question. Is $q \psi \leqq q X$ for any tree-like continuum $X$ ?

The appropriate modifications of Lemma 4.1, and Theorems 4.3 and 4.4 yield the following three results.

Lemma 4.3. If $X$ is a linear graph, then $\gamma$-ord $X \leqq 3$.

THEOREM 4.5. A continuum $X$ is 1-dimensional if and only if $\gamma$-ord $X \leqq 3$.

THEOREM 4.6. A continuum $X$ is 1-dimensional if and only if $q X \leqq q \mathfrak{M}$.

From the results of $\S 3$ we obtain the next two theorems.

Theorem 4.7. If $X$ is a Peano curve which does not contain a primitive skew curve, then

(1) $q I \leqq q X \leqq q \subseteq$;

(2) $X$ is an arc if and only if $q X \leqq q I$.

COROLLARY. If $X$ is a continuum with coverings of arbitrarily small mesh whose nerves are planar linear graphs (i.e., do not contain a primitive skew curve), then $q X \leqq q \subseteq\left(<q R^{2}\right)$. (For example, tree-like continua have such coverings.)

Theorem 4.8. If $X$ is a Peano curve which contains a primitive skew curve, then

(1) $q X \not q R^{2}$;

( 2) $\left\{\begin{array}{l}q K_{1} \\ q K_{2}\end{array}\right\} \leqq q X \leqq q \mathfrak{M}$;

(3) $X$ is homeomorphic to $K_{1}$ if and only if $q X=q K_{1}$;

(4) $X$ is homeomorphic to $K_{2}$ if and only if $q X=q K_{2}$.

Proof. We consider only one implication from each of (3) and (4) (in that order). Assuming that $q X=q K_{1}$ by Theorem 3.7 we have that $X$ contains $K$, a topological image of $K_{1}$. So the $\varepsilon$-mappings of $X$ into $K_{1}$ must take $K$ onto all of $K_{1}$ for $\varepsilon$ sufficiently small. So we must have that $X=K$.

Assuming that $q X=q K_{2}$ we have by Theorem 3.8 that $X$ contains $K$, a topological image of $K_{1}$ or $K_{2}$. The former case is impossible since $K_{1}$ and $K_{2}$ are not comparable with respect to quasi dimension type. So $\varepsilon$-mappings of $X$ into $K_{2}$ must take $K$ onto all of $K_{2}$ for $\varepsilon$ sufficiently small. So we must have that $X=K$.

Added in proof. I intended to construct a snake-like continuum with $2^{c}$ different quasi dimension types among its subsets. However, this and the two questions in $\S 1$ remain unsettled. The situation as regards circle-like continua is clearer. H. Cook [Fund. Math. 60 (1967) 
241-249] has constructed a 1-dimensional continuum $M_{1}$ containing $c$ continua which are circle-like but not snake-like and no one which is the continuous image of the other. It follows that no two of these circle-like continua are comparable with respect to quasi dimension type. Furthermore, since there are at most $c$ topologically different circle-like continua, we may conclude that the cardinality of the set of quasi dimension types of circle-like continua is $\boldsymbol{c}$.

\section{BIBLIOGRAPHY}

1. R. H. Bing, Concerning hereditarily indecomposable continua, Pacific J. Math. 1 (1951), 43-51.

2. - A homogeneous indecomposable plane continuum, Duke Math. J. 15 (1948), 729-742.

3. The simple connectivity of the sum of two disks, Pacific J. Math. 14 (1964), 439-455.

4. R. H. Bing and F. B. Jones, Another homogeneous plane continuum, Trans. Amer. Math. Soc. 90 (1959), 171-192.

5. — Snake-like continua, Duke Math. J. 18 (1951), 653-663.

6. S. Claytor, Topological immersion of peanian continuum in a spherical surface, Ann. of Math. 35 (1934), 809-835.

7. - Peanian continua not imbeddable in a spherical surface, Ann. of Math. 38 (1937), 631-646.

8. H. Cook, On the most general plane closed point set through which it is possible to pass a pseudo-arc, Fund. Math. 55 (1964), 11-22.

9. H. H. Corson and J. R. Isbell, Some properties of strong uniformities, Quar. J. Math. (2) 11 (1960), 17-33.

10. M. K. Fort, Images of plane continua, Amer. J. Math. 81 (1959), 541-546.

11. T. Ganea, Comment on the imbedding of polyhedra in Euclidean spaces, Bull. Acad. Polon. Sci. 7 (1959), 27-32.

12. W. Hurewicz and H. Wallman, Dimension Theory, Princeton, 1948.

13. J. R. Isbell, Embeddings of inverse limits, Ann. of Math. 70 (1959), 73-84.

14. J. L. Kelley, Hyperspaces of a continuum, Trans. Amer. Math. Soc. 52 (1942), $22-36$.

15. C. Kuratowski, Sur le problème des courbes gauches en Topologie, Fund. Math. 15 (1930), 271-283.

16. C. Kuratowski and S. M. Ulam, Sur un coefficient lie aux transformatuns continues d'ensembles, Fund. Math. 20 (1933), 244-253.

17. A. Lelek, On weakly chainable continua, Fund. Math. 51 (1962), 271-282.

18. S. Mazurkiewicz, Uber nicht plättbare Kurven, Fund. Math. 20 (1933), 281-284.

19. K. Menger, Kurventheorie, Berlin, 1932.

20. — Zur allgeneinen Kurventheorie, Fund. Math. 10 (1927), 96-115.

21. J. Segal, Quasi dimension type. I., Pacific J. Math. 20 (1967), 501-534.

22. - Mapping norms and indecomposability, Lond. J. Math. 39 (1964), 598-602.

23. H. Whitney, Regular families of curves, Ann. of Math. (2) 34 (1933), 244-270.

24. G. T. Whyburn, A continuum every subcontinuum of which separates the plane, Amer. J. Math. 52 (1930), 319-330.

Received October 26, 1964, in revised form August 2, 1967.

UNIVERSITY OF WASHINGTON

Seattle, Washington 


\section{PACIFIC JOURNAL OF MATHEMATICS}

\section{EDITORS}

\section{H. ROYDEN}

Stanford University

Stanford, California
J. DugundJI

Department of Mathematics

Rice University

Houston, Texas 77001

RICHARD ARENS

University of California

Los Angeles, California 90024

Seattle, Washington 98105

\section{ASSOCIATE EDITORS}
E. F. BeCKENBACH
B. H. NeumanN
F. WOLF
K. YOSIDA

\section{SUPPORTING INSTITUTIONS}

\author{
UNIVERSITY OF BRITISH COLUMBIA \\ CALIFORNIA INSTITUTE OF TECHNOLOGY \\ UNIVERSITY OF CALIFORNIA \\ MONTANA STATE UNIVERSITY \\ UNIVERSITY OF NEVADA \\ NEW MEXICO STATE UNIVERSITY \\ OREGON STATE UNIVERSITY \\ UNIVERSITY OF OREGON \\ OSAKA UNIVERSITY \\ UNIVERSITY OF SOUTHERN CALIFORNIA
}

\author{
STANFORD UNIVERSITY \\ UNIVERSITY OF TOKYO \\ UNIVERSITY OF UTAH \\ WASHINGTON STATE UNIVERSITY \\ UNIVERSITY OF WASHINGTON \\ * * * * \\ AMERICAN MATHEMATICAL SOCIETY \\ CHEVRON RESEARCH CORPORATION \\ TRW SYSTEMS \\ NAVAL WEAPONS CENTER
}

Mathematical papers intended for publication in the Pacific Journal of Mathematics should be in typed form or offset-reproduced, double spaced with large margins. Underline Greek letters in red, German in green, and script in blue. The first paragraph or two must be capable of being used separately as a synopsis of the entire paper. It should not contain references to the bibliography. Manuscripts, in duplicate if possible, may be sent to any one of the four editors. All other communications to the editors should be addressed to the managing editor, Richard Arens, University of California, Los Angeles, California 90024.

Each author of each article receives 50 reprints free of charge; additional copies may be obtained at cost in multiples of 50 .

The Pacific Journal of Mathematics is published monthly. Effective with Volume 16 the price per volume (3 numbers) is $\$ 8.00$; single issues, $\$ 3.00$. Special price for current issues to individual faculty members of supporting institutions and to individual members of the American Mathematical Society: $\$ 4.00$ per volume; single issues $\$ 1.50$. Back numbers are available.

Subscriptions, orders for back numbers, and changes of address should be sent to Pacific Journal of Mathematics, 103 Highland Boulevard, Berkeley 8, California.

Printed at Kokusai Bunken Insatsusha (International Academic Printing Co., Ltd.), 7-17, Fujimi 2-chome, Chiyoda-ku, Tokyo, Japan.

PUBLISHED BY PACIFIC JOURNAL OF MATHEMATICS, A NON-PROFIT CORPORATION

The Supporting Institutions listed above contribute to the cost of publication of this Journal, but they are not owners of publishers and have no responsibility for its content or policies. 


\section{Pacific Journal of Mathematics \\ Vol. 25, No. $2 \quad$ October, 1968}

Martin Aigner, On the tetrahedral graph ..................... 219

Gregory Frank Bachelis, Homomorphisms of annihilator Banach

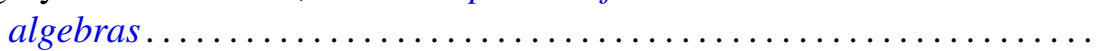

Phillip Alan Griffith, Transitive and fully transitive primary abelian groups.......................................... 249

Benjamin Rigler Halpern, Fixed points for iterates . . . . . . . . . . . . . 255

James Edgar Keesling, Mappings and dimension in general metric spaces ......................................... 277

$\mathrm{Al}$ (Allen Frederick) Kelley, Jr., Invariance for linear systems of ordinary differential equations ................................ 289

Hayri Korezlioglu, Reproducing kernels in separable Hilbert spaces . . . . . 305

Gerson Louis Levin and Wolmer Vasconcelos, Homological dimensions and Macaulay rings ................................. 315

Leo Sario and Mitsuru Nakai, Point norms in the construction of harmonic

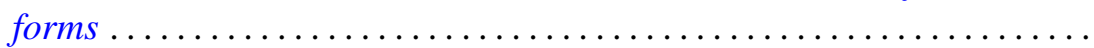

Barbara Osofsky, Noncommutative rings whose cyclic modules have cyclic injective hulls ..................................... 331

Newton Tenney Peck, Extreme points and dimension theory........... 341

Jack Segal, Quasi dimension type. II. Types in 1-dimensional spaces ...... 353

Michael Schilder, Expected values of functionals with respect to the Ito distribution ...

Grigorios Tsagas, A Riemannian space with strictly positive sectional curvature

John Alexander Williamson, Random walks and Riesz kernels . . 